\title{
Metrization of Weakly Developable Spaces
}

\author{
Abdul M. Mohamad \\ Department of Mathematics and Statistics, College of Science, Sultan Qaboos \\ University, P.O. Box 36, Al Khod 123, Muscat, Sultanate of Oman, \\ Email:mohamad@squ.edu.om.

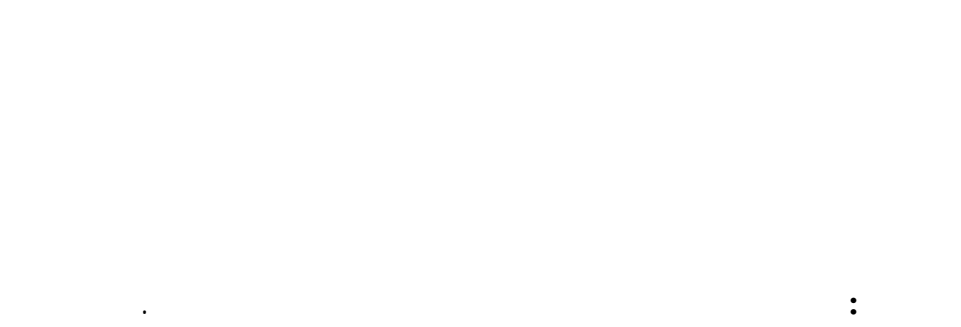

ABSTRACT: In this note, we present metrization of weak developability.

Keywords: Weakly Developable, Metrizable, Weak Base.

\section{Introduction}

$\mathbf{M}$

artin (1976) introduced the concept of weak developability in order to study the problem of the metrization of spaces with weak bases. A space $X$ is weakly developable if and only if there is a sequence $\left\{G_{n}\right\}_{n \in N}$ of covers of $X$ such that for each $x \in X,\left\{s t\left(x, G_{n}\right): n \in N\right\}$ is a weak base at $x$. The sequence $\left\{G_{n}\right\}_{n \in N}$ is said to be a weak development for the space $X$. If each $G_{n}$ consists of open sets, then $\left\{G_{n}\right\}_{n \in N}$ is a development for the space $X$ and $X$ is a developable space (Gruenhage, 1984).

The idea of weak base was introduced by Arhangel'skii (1966) in the study of symmetrizable spaces. It is more convenient to use the form of Siwiec, (1974) and Franklin, (1965).

A collection $w$ of subsets of a space $X$ is called a weak base for $X$ provided that to each $x \in X$, there exists $w_{x} \subset w$ such that

1. Each member of $w_{x}$ contains $x$.

2. For any two members $W_{1}$ and $W_{2}$ of $w_{x}$, there is a $W_{3}$ in $w_{x}$, such that $W_{3} \subset W_{1} \cap W_{2}$. 3. A subset $F$ of $X$ is closed if and only if for every point $x \notin F$, there exists a $W$ in $w_{x}$ such that $F \cap W=\phi$.

If to each $x \in X$ we assign a collection $w_{x}$ of supersets of $\{x\}$ such that $W=\cup\left\{w_{x}: x \in X\right\}$ is a weak base by virtue of the collections $w_{x}$, i.e., the collections $w_{x}$ satisfy conditions (1), (2) and (3) of the preceding paragraph, then we say that the collection $w_{x}$ is a local weak base at $x$ for each $x \in X$. It is easy to show that a subset $O$ of a space $X$ with local weak bases $\left\{w_{x}: x \in X\right\}$ is open if and only if for each $x \in O$, there is a member $W$ of the local weak base $w_{x}$ of $x \in X$ with $W \subset O$.

In this study, we prove a metrization theorem for weakly developable spaces. We assume throughout this note that all spaces are $T_{0}$. A topological space is a $T_{0}$-space if, and only if, for each pair $x$ and $y$ of distint points, there is a nighborhood of one point to which the other does not 
belong. Also, we let $N$ denote the set of all positive integers. For a collection $G$ of subsets of a space $X$, we define $s t(x, G)=\cup\{g: x \in g \in G\}$ and $s t^{2}(x, G)=\cup\{s t(y, G): y \in s t(x, G)\}$.

\section{Main Results}

\section{1 lemma 1}

Let $\left\{G_{n}\right\}_{n \in N}$ be a weak development of a space $X$. Then for every compact subset $K$ of $X$ and any sequence $\left\langle y_{n}: n \in N\right\rangle$ of points in $K$, there is a point $y$ in $X$ and a subsequence $\left\langle y_{n}: i \in N\right\rangle$ of the sequences $\left\langle y_{n}: n \in N\right\rangle$ which converges to $y$.

Proof. Let $K$ be a compact subset of $X$ and let $\left\langle y_{n}: n \in N\right\rangle$ be any sequence of points in $K$. Suppose there is no subsequence of $\left\langle y_{n}: n \in N\right\rangle$ which converges to a point of $X-\left\{y_{n}: n \in N\right\}$. Then, we note that $F=\left\{y_{n}: n \in N\right\}$ is a closed subset of $X$. For if it is not true then for some point $y \in X-F$ we shall have $y_{n_{1}} \in \operatorname{st}\left(y, G_{i}\right) \cap F$ for each $i \in N$. This will imply that the subsequence $\left\langle y_{n}: i \in N\right\rangle$ will converge to $y$, which will contradict our assumption. Therefore, $F$ is closed.

Define $F_{n}=\left\{y_{i}: i \geq n\right\}$ for each $n \in N$. Similarly, one can show that $F_{n}$ is closed for each $n \in N$. Consider the open cover $\left\{X-F_{n}: n \in N\right\}$ of $K$ in $X$. It is easy to see that it does not contain a finite subcover of $K$ which contradicts the fact that $K$ is compact. Hence, the sequences $\left\langle y_{n}: n \in N\right\rangle$ have a convergent subsequence.

\section{2 lemma 2}

Let $\left\{G_{n}\right\}_{n \in N}$ be a weak development of a space $X$, which satisfies the following condition: For any closed subset $F$ of $X$ and any point $y \in X-F$, there is an $n \in N$ such that $s t\left(y, G_{n}\right) \cap s t\left(F, G_{n}\right)=\phi$. Then every compact subset of $X$ is closed.

Proof. Let $K$ be a compact subset of $X$. Suppose $K$ is not closed. Then there is a point $y \in X-K$ such that $s t\left(y, G_{i}\right) \cap K \neq \phi$ for all $i \in N$. Thus for each $i \in N$, let $y_{i} \in \operatorname{st}\left(y, G_{i}\right) \cap K$. Hence, the sequence $\left\langle y_{i}: i \in N\right\rangle$ converges to $y$. Put $F=\left\{y_{i}: i \in N\right\} \cup\{y\}$. We claim that $F$ is a closed subset of $X$. For if not, then there is a point $z \in X-F$ such that $s t\left(z, G_{i}\right) \cap F \neq \phi$ for all $i \in N$. Without loss of generality, let $y_{i} \in S t\left(z, G_{i}\right) \cap F$ for all $i \in N$. This is not possible since a weakly developable space is $T_{1}$ and hence by the hypothesis there is an $n \in N$ such that $\operatorname{st}\left(z, G_{n}\right) \cap \operatorname{st}\left(y, G_{n}\right)=\phi$. Define $F_{n}=\left\{y_{i}: i \geq n\right\} \cup\{y\}$ for each $n \in N$. Clearly, $F_{n}$ is closed for each $n \in N$. Therefore, $\left\{X-F_{n}: n \in N\right\}$ is an open cover of $K$ with no finite subcover of $K$ giving a contradiction.

\subsection{Theorem}

The following are equivalent for a space $X$.

1 . The space $X$ is metrizable.

2. The space $X$ has a weak development $\left\{G_{n}\right\}_{n \in N}$ such that for any closed subset $F$ of $X$ and any point $x \in X-F$, there is an $i \in N$ such that $s t\left(y, G_{i}\right) \cap s t\left(F, G_{i}\right)=\phi$ 
3. The space $X$ has a weak development $\left\{G_{n}\right\}_{n \in N}$ such that if $A \subset V$, where $A$ is compact and $V$ is open, then $s t\left(A, G_{n}\right) \subset V$ for some $n$.

4. The space $X$ has a weak development $\left\{G_{n}\right\}_{n \in N}$ such that if $x \in V$, where $V$ is open, then there exists a neighborhood $U$ of $x$ and $n \in V$, for which $s t\left(U, G_{n}\right) \subset V$.

5. The space $X$ has a weak development $\left\{G_{n}\right\}_{n \in N}$ such that if $x \in V$ is open, then there exists an $n \in N$ for which $s t^{2}\left(x, G_{n}\right) \subset V$.

Proof. The implication $1 \Rightarrow 2$ is clear. The implications $3 \Rightarrow 4,4 \Rightarrow 5$ and $5 \Rightarrow 1$ are proved in Martin (1976, Theorem 2.5 and Theorem 2.6). To prove $2 \Rightarrow 3$. Let $A$ be any compact subset of $X$ and let $V$ be an open subset of $X$ containing $A$. Suppose that $\operatorname{st}\left(A, G_{i}\right) \cap(X-U) \neq \phi$. Let $x \in \operatorname{st}\left(A, G_{i}\right) \cap(X-V)$. Hence, by Lemmas 2.1 and 2.2 there is a subsequence $\left\langle x_{i_{k}}: k \in \mathrm{N}\right\rangle$ of the sequence $\left\langle x_{i}: i \in \mathrm{N}\right\rangle$, which converges to a point $y$ in $A$. Now, by hypothesis there is a $j \in \mathrm{N}$ such that $s t\left(y, G_{i}\right) \cap s t\left((X-V), G_{i}\right)=\phi$. This leads to a contradiction.

\section{Acknowledgement}

The author is grateful to Prof. David Gauld for his kind help and suggestions on this paper.

\section{References}

ARHANGEL'SKII, A. 1966. Mappings and spaces, Russian Math. Surveys, 21:115--162. FRANKLIN, S. 1965. Spaces in which sequences suffice. II, Fund. Math. 57:107-115. GRUENHAGE, G. 1984. Generalized metric spaces, in Handbook of Set-theoretic Topology, pp. 423-501.

MARTIN, H. 1976. Weak bases and metrization, Trans. Amer. Math. Soc. 222: 338-344.

SIWIEC, F. 1974. On defining a space by a weak base, Pacific J. Math. 52:233-245.

Received 14 May 2001

Accepted 6 June 2002 